07,01

\title{
Влияние водорода на модуль Юнга и внутреннее трение сплава V-4Ti-4Cr
}

\author{
(C) Б.К. Кардашев, К.В. Сапожников \\ Физико-технический институт им. А.Ф. Иофрфе РАН, \\ Санкт-Петербург, Россия \\ ฯ E-mail: k.sapozhnikov@mail.ioffe.ru \\ Поступила в Редакцию 20 июля 2021 г. \\ В окончательной редакции 20 июля 2021 г. \\ Приянта к публикации 23 июля 2021 г.
}

\begin{abstract}
Модуль Юнга и внутреннее трение сплава V-4Ti-4Cr с различной концентрацией примеси водорода исследованы в интервале температур $100-300 \mathrm{~K}$ при частотах колебаний около $100 \mathrm{kHz}$. Показано, что наводороживание сплава приводит к появлению минимума на температурной зависимости модуля Юнга вследствие выпадения/растворения частиц моногидрида ванадия, и релаксационного пика в температурном спектре внутреннего трения при температурах около $140 \mathrm{~K}$, который можно связать с водородной релаксацией типа релаксации Снука, обусловленной взаимодействием атомов водорода с атомами Тi.
\end{abstract}

Ключевые слова: сплав V-4Ti-4Cr, водород, внутреннее трение, модуль Юнга, неупругие эффекты.

DOI: 10.21883/FTT.2021.12.51676.171

\section{1. Введение}

Сплавы системы V-Ti-Cr с объемно-центрированной кубической решеткой являются перспективными конструкционными материалами для активных зон реакторов термоядерного синтеза и реакторов деления на быстрых нейтронах [1-4]. В процессе эксплуатации подразумевается контакт этих сплавов с внешней газовой средой, включающей такие компоненты (например, водород и дейтерий), которые могут проникать внутрь сплава и менять его свойства. В литературе имеются данные по влиянию водорода на механические свойства сплавов V-Ti-Cr при растяжении [5-9] и ударном нагружении [9]. Однако вопрос о влиянии водорода на упругие и неупругие свойства сплавов системы V-Ti-Cr до сих пор не изучался. Данная работа на примере образцов сплава, содержащего по 4 at.\% титана и хрома (V-4Ti-4Cr) и различные концентрации примеси водорода в какой-то степени восполняет этот пробел.

\section{2. Образцы и методика эксперимента}

Исследования были проведены на сплаве V-4Ti-4Cr, изготовленном по технологии, разработанной в АО „ВНИИНМ им. А.А Бочвара““ [2]. Содержание основных компонентов и примесей в сплаве, wt.\%: Тi - 4.36; $\mathrm{Cr}-4.21 ; \mathrm{B}-0.002 ; \mathrm{C}-0.013 ; \mathrm{N}-0.011 ; \mathrm{O}-$ 0.02; $\mathrm{Al}-0.005 ; \mathrm{Si}-0.019 ; \mathrm{Fe}-0.0084$ [2]. Образцы для акустических измерений имели форму стержней прямоугольного сечения размерами $1 \times 5 \mathrm{~mm}$ и длиной около $23 \mathrm{~mm}$. Такая длина обеспечивала резонансную частоту продольных колебаний образцов вблизи $100 \mathrm{kHz}$. Образцы насыщались водородом до различных концентраций в водородной атмосфере методом Сивертса.
Результаты измерений наводороженных образцов сопоставляются с данными для образца, не подвергавшегося наводороживанию.

Акустический резонансный метод составного пьезоэлектрического вибратора использовался для регистрации температурных зависимостей модуля Юнга и внутреннего трения образцов в интервале температур $100-300$ K. Подробное описание метода приведено в [10]. В качестве меры внутреннего трения использовался логарифмический декремент колебаний $\delta$. Модуль Юнга $E$ образцов определялся из их плотности $\rho$, длины $l$ и частоты основной гармоники $f: E=4 \rho f^{2} l^{2}$. Амплитуда колебательной деформации $\varepsilon_{m}$ автоматически поддерживалась постоянной на уровне $10^{-6}$, соответствующем области амплитудно-независимого внутреннего трения.

\section{3. Экспериментальные результаты}

Типичные результаты измерений модуля Юнга и внутреннего трения сплава $\mathrm{V}-4 \mathrm{Ti}-4 \mathrm{Cr}$ в термоцикле охлаждение-нагрев представлены на рис. 1 на примере исходного (не подвергавшегося наводороживанию) образца и образца, содержащего 4.9 at.\% водорода. В исходном образце в исследованном диапазоне температур наблюдается низкий уровень внутреннего трения и отсутствие каких-либо особенностей на температурных зависимостях внутреннего трения и модуля Юнга. В наводороженном образце при температурах около $140 \mathrm{~K}$ наблюдается максимум внутреннего трения. Величина модуля Юнга у наводороженного образца существенно ниже, чем у исходного, а на его температурной зависимости наблюдаются минимум при температурах около $180 \mathrm{~K}$ и температурный гистерезис, сильнее всего 
выраженный вблизи минимума. Отметим, что небольшой температурный гистерезис наблюдается также для внутреннего трения: при температурах $150-180 \mathrm{~K}$ внутреннее трение несколько выше при охлаждении, чем при нагреве.

На рис. 2 показано влияние концентрации водорода в образцах сплава V-4Ti-4Cr на температурные зависимости модуля Юнга и внутреннего трения, измеренные при нагреве. Видно, что минимум на температурной зависимости модуля Юнга сдвигается к более высоким температурам при увеличении концентрации водорода. В то же время величина и температурное положение максимума на температурной зависимости внутреннего трения не зависят от концентрации водорода в исследованном диапазоне концентраций.

\section{4. Обсуждение}

Известно, что присутствие водорода в ванадии и его сплавах приводит к целому ряду неупругих эффектов [11]: 1) эффекты, связанные с выпадением и растворением гидридов; 2) водородная релаксация типа релаксации Снука, обусловленная взаимодействием водорода с примесными атомами внедрения или замещения; 3) водородная релаксация Снука-Кёстера.

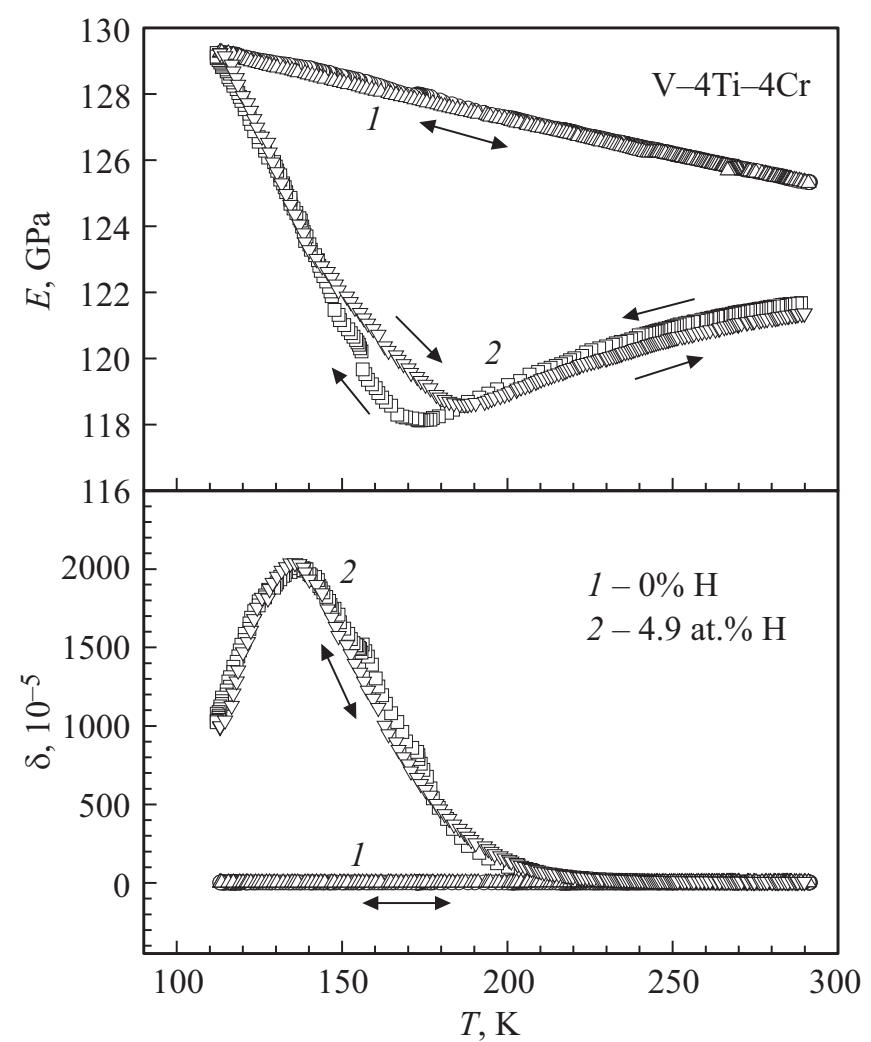

Рис. 1. Температурные зависимости модуля Юнга $E(a)$ и декремента $\delta(b)$ исходного образца $(1)$ и образца, содержащего 4.9 at.\% водорода (2), сплава V-4Ti-4Cr. Стрелки указывают направление изменения температуры.

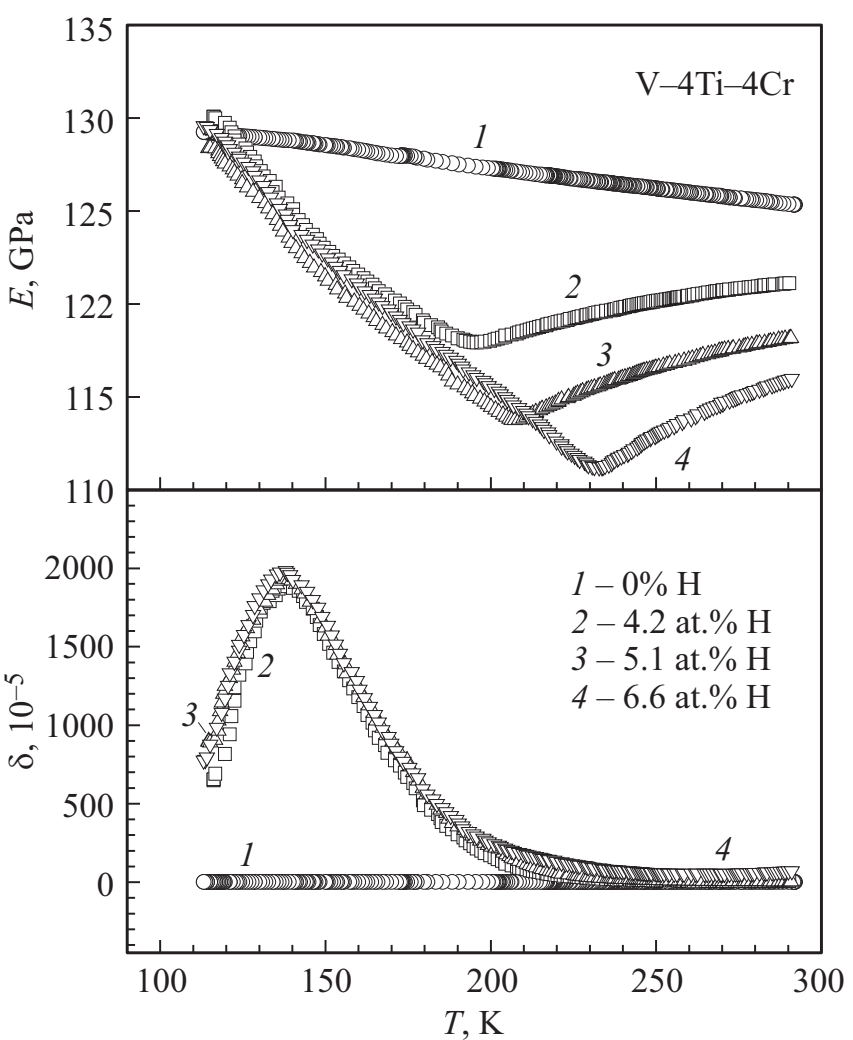

Рис. 2. Температурные зависимости модуля Юнга $E(a)$ и декремента $\delta(b)$ исходного образца $(1)$ и образцов, насыщенных водородом $(2,3,4)$, сплава V-4Ti-4Cr, измеренные в процессе нагрева.

Ванадий является гидридообразующим металлом. Зависимость растворимости водорода в металле от температуры приводит при термоциклировании к обратимым процессам выпадения и растворения частиц гидридов. Эти процессы обычно сопровождаются появлением асимметричного нерелаксационного максимума в температурном спектре внутреннего трения. В системе V-H этот эффект хорошо исследован для случая моногидрида V [12-21]. Показано, что максимум внутреннего трения состоит из двух компонент: переходной (transient), пропорциональной скорости изменения температуры и обратно пропорциональной частоте колебаний, и равновесной, не зависящей от скорости изменения температуры $[11,17,21]$. Максимум внутреннего трения сопровождается минимумом модуля упругости $[16,21]$.

Наличие межузельного водорода не приводит к классической релаксации Снука в чистых металлах с объемно-центрированной кубической решеткой вследствие исчезающе малой мощности упругих диполей [11]. В то же время захват межузельного водорода примесями, как межузельными, так и примесями замещения, может приводить к релаксации типа релаксации Снука, вследствие образования упругих диполей водородпримесный атом [11]. В ванадии такие релаксации были 
обнаружены для примесей внедрения О и $\mathrm{N}[18,20]$, а также примесей замещения $\mathrm{Ti}$ и $\mathrm{Zr}[22,23]$.

Водородная релаксация Снука-Кёстера представляет собой дислокационную релаксацию, контролируемую диффузией межузельных атомов водорода, захваченных дислокациями [11]. Эта релаксация обусловливает появление релаксационного максимума в температурных спектрах внутреннего трения деформированного ванадия $[12,15,16,24,25]$. В литературе этот максимум зачастую фигурирует как $\alpha$ пик (так обычно обозначается собственно дислокационная релаксация невинтовых дислокаций в металлах с объемно-центрированной кубической решеткой [11]), поскольку роль водорода в его появлении была выяснена не сразу.

Рассмотрим наблюдающиеся в наводороженном сплаве V-4Ti-4Cr неупругие эффекты. Минимум на температурной зависимости модуля Юнга, температурное положение которого зависит от концентрации водорода, можно связать с процессами выпадения и растворения частиц моногидрида ванадия. Отсутствие соответствующего максимума внутреннего трения объясняется тем, что обе компоненты внутреннего трения, сопровождающие процессы выпадения и растворения частиц, в нашем случае малы. Переходная компонента внутреннего трения, обратно пропорциональная частоте колебаний, пренебрежимо мала при частотах колебаний порядка $10^{5} \mathrm{~Hz}$. Равновесная компонента внутреннего трения в системе $\mathrm{V}-\mathrm{H}$ также убывает с ростом частоты колебаний $[17,20]$, становясь незначительной в килогерцевом диапазоне частот [20,21]. Отметим слабый гистерезис на спектре внутреннего трения в окрестностях температур минимума модуля Юнга, указывающий на следы равновесной компоненты ВТ, сопровождающей выпадение и растворение частиц гидрида (рис. 1).

Поведение модуля Юнга в окрестностях максимума внутреннего трения, наблюдающегося в наводороженных образцах при температурах около $140 \mathrm{~K}$, указывает на релаксационную природу максимума. Этот максимум не может быть связан с водородной релаксацией Снука-Кёстера. Оценки значения температуры максимума Снука-Кёстера для частоты колебаний $100 \mathrm{kHz}$ по имеющимся литературным данным показывают, что его следует ожидать при температурах 200-270 К. На наших спектрах внутреннего трения никаких особенностей в этом диапазоне температур нет. Отсутствие релаксации Снука-Кёстера в исследованных образцах объясняется тем, что для ее наблюдения требуется пластическая деформация образцов. В нашем случае образцы не подвергались предварительной пластической деформации, а аккомодация объемных изменений при выпадении частиц гидридов в системе V-H происходит преимущественно упруго вследствие их малости [26], и для появления пика Снука-Кёстера требуется большое число термоциклов (см. рис. 7 [16]). Кроме того, процессы пластической аккомодации при выпадении и растворении частиц гидридов дополнительно подавляются в исследуемом сплаве атомами Тi и $\mathrm{Cr}$.
Оценка значений температур максимумов внутреннего трения для водородных релаксаций типа релаксации Снука по имеющимся данным для пар $\mathrm{O}(\mathrm{N})-\mathrm{H}[18]$ и $\mathrm{Ti}-\mathrm{H}[22,23]$ дает для частоты колебаний $100 \mathrm{kHz}$ значения около 100 и $116 \mathrm{~K}$ соответственно. Величина наблюдаемого нами пика внутреннего трения очень близка величине пика, обусловленного релаксацией упругих диполей Тi-H в сплаве V-3.9at.\%Ti-4.44at.\%H [22,23]. Концентрация примесей $\mathrm{O}$ и $\mathrm{N}$ в наших образцах гораздо ниже концентрации атомов Ті. Кроме того, по данным работы [27], примеси О и $\mathrm{N}$ в сплаве $\mathrm{V}-4 \mathrm{Ti}-4 \mathrm{Cr}$ находятся не в твердом растворе, а в связанном состоянии. Отметим также, что атомы $\mathrm{Cr}$, в отличие от атомов $\mathrm{Ti}$, не являются ловушками для атомов водорода в ванадии [28]. Таким образом, наблюдаемый в наводороженных образцах максимум внутреннего трения можно связать с водородной релаксацией типа релаксации Снука, обусловленной взаимодействием атомов водорода с атомами Ті. Независимость величины пика внутреннего трения от концентрации водорода в наших данных (рис. 2) можно связать с тем, что измерения проводились при концентрациях, соответствующих насыщению количества пар Тi-H.

\section{Благодарности}

Авторы выражают благодарность В.М. Чернову (АО „ВНИИНМ им. А.А. Бочвара“) за предоставленные для исследования образцы.

\section{Конфликт интересов}

Авторы заявляют, что у них нет конфликта интересов.

\section{Список литературы}

[1] R.J. Kurtz, K. Abe, V.M. Chernov, D.T. Hoelzer, H. Matsui, T. Muroga, G.R. Odette. J. Nucl. Mater. 329-333, 47 (2004).

[2] А.К. Шиков, В.М. Чернов, М.М. Потапенко, И.Н. Губкин, В.А. Дробышев, В.С. Зурабов. МиТОМ 11, 49 (2004).

[3] J.M. Chen, V.M. Chernov, R.J. Kurtz, T. Muroga. J. Nucl. Mater. 417, 289 (2011).

[4] T. Muroga, J.M. Chen, V.M. Chernov, R.J. Kurtz, M. Le Flem. J. Nucl. Mater. 455, 263 (2014).

[5] J.R. DiStefano, J.H. De Van, D.H. Röhrig, L.D. Chitwood. J. Nucl. Mater. 273, 102 (1999).

[6] K. Natesan, W.K. Soppet. J. Nucl. Mater. 283-287, 1316 (2000).

[7] K. Aoyagi, E.P. Torres, T. Suda, S. Ohnuki. J. Nucl. Mater. 283-287, 876 (2000)

[8] J. Chen, Z. Xu, L. Yang. J. Nucl. Mater. 307-311, 566 (2002).

[9] J. Chen, T. Muroga, S. Qiu, Y. Xu, Y. Den, Z. Xu. J. Nucl. Mater. 325, 79 (2004).

[10] С.П. Никаноров, Б.К. Кардашев. Упругость и дислокационная неупругость кристаллов. Наука, М. (1985) 254 с.

[11] M.S. Blanter, I.S. Golovin, H. Neuhäuser, H.-R. Sinning. Internal Friction in Metallic Materials. A Handbook. SpringerVerlag, Berlin-Heidelberg (2007). 539 p. 
[12] G. Cannelli, F.M. Mazzolai. J. Phys. Chem. Solids 31, 1913 (1970).

[13] C.V. Owen, T.E. Scott. Met. Mater. Trans. B 3, 1715 (1972).

[14] H.Y. Chang, C.A. Wert. Acta Met. 21, 1233 (1973).

[15] M. Koiwa, K. Shibata, O. Yoshinari. Trans. JIM 21, 191 (1980).

[16] O. Yoshinari, M. Koiwa. Acta Met. 30, 1979 (1982).

[17] O. Yoshinari, M. Koiwa. Acta Met. 30, 1987 (1982).

[18] G. Cannelli, R. Cantelli, F. Cordero. J. Phys. (Fr.) 44 (Coll. C9), 403 (1983).

[19] C.V. Owen, O. Buck. Scripta Met. 17, 649 (1983).

[20] G. Cannelli, R. Cantelli, F. Cordero. Scripta Met. 18, 1031 (1984).

[21] M. Koiwa, O. Yoshinari. J. Phys. (Fr.) 46 (Coll. C10), 99 (1985).

[22] S. Tanaka, M. Koiwa. J. Phys. (Fr.) 42 (Coll. C5), 781 (1981).

[23] S. Tanaka, M. Koiwa. Scripta Met. 15, 403 (1981).

[24] H. Mizubayashi, S. Okuda, M. Daikubara. Scripta Met. 13, 1131 (1979).

[25] H. Mizubayashi, S. Amano, S. Okuda, M. Shimada. Scripta Met. 16, 343 (1982).

[26] B.J. Makenas, H.K. Birnbaum. Acta Met. 28, 979 (1980).

[27] К.А. Мороз, В.М. Чернов, М.М. Потапенко, В.А. Дробышев, М.В. Кравцова. Перспективные материалы 3, 14 (2020).

[28] S. Tanaka, H. Kimura. Trans. JIM 20, 647 (1979).

Редактор К.В. Емцев 